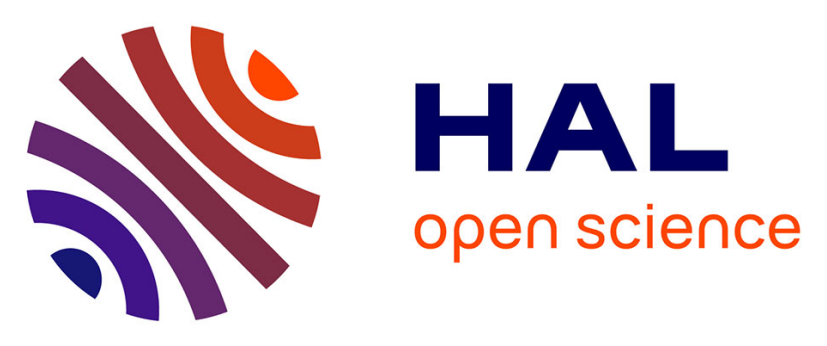

\title{
Injury to rat carotids causes time-dependent changes in gene expression in contralateral uninjured arteries
}

Amalia Forte, Mauro Finicelli, Pasquale de Luca, Ina Nordstrom, Francesco

Onorati, Cesare Quarto, Pasquale Santè, Attilio Renzulli, Umberto Galderisi, Liberato Berrino, et al.

\section{To cite this version:}

Amalia Forte, Mauro Finicelli, Pasquale de Luca, Ina Nordstrom, Francesco Onorati, et al.. Injury to rat carotids causes time-dependent changes in gene expression in contralateral uninjured arteries. Clinical Science, 2008, 116 (2), pp.125-136. 10.1042/CS20080080 . hal-00479431

\section{HAL Id: hal-00479431 \\ https://hal.science/hal-00479431}

Submitted on 30 Apr 2010

HAL is a multi-disciplinary open access archive for the deposit and dissemination of scientific research documents, whether they are published or not. The documents may come from teaching and research institutions in France or abroad, or from public or private research centers.
L'archive ouverte pluridisciplinaire HAL, est destinée au dépôt et à la diffusion de documents scientifiques de niveau recherche, publiés ou non, émanant des établissements d'enseignement et de recherche français ou étrangers, des laboratoires publics ou privés. 


\title{
INJURY TO RAT CAROTIDS CAUSES TIME-DEPENDENT CHANGES IN GENE EXPRESSION IN CONTRALATERAL UNINJURED ARTERIES
}

\author{
${ }^{* 1}$ Forte Amalia, ${ }^{1}$ Finicelli Mauro, ${ }^{5}$ De Luca Pasquale, ${ }^{4}$ Nordström Ina, ${ }^{3}$ Onorati Francesco, ${ }^{2}$ Quarto \\ Cesare, ${ }^{2}$ Santè Pasquale, ${ }^{3}$ Renzulli Attilio ${ }^{1}$ Galderisi Umberto, ${ }^{1}$ Berrino Liberato, ${ }^{2}$ De Feo Marisa, \\ ${ }^{4}$ Hellstrand Per, ${ }^{1}$ Rossi Francesco, ${ }^{2}$ Cotrufo Maurizio, ${ }^{1}$ Cascino Antonino, ${ }^{1}$ Cipollaro Marilena
}

Excellence Research Centre for Cardiovascular Diseases, Departments of ${ }^{1}$ Experimental Medicine and ${ }^{2}$ Cardiothoracic Sciences, Second University of Naples, Italy; ${ }^{3}$ Unit of Cardiac Surgery, University Magna Graecia, Catanzaro, Italy; ${ }^{4}$ Department of Experimental Medical Science, Lund University, Sweden; ${ }^{5}$ BIOGEM S.c.a.r.l., Ariano Irpino, Italy

\section{*Correspondence to:}

Amalia Forte

Department of Experimental Medicine,

Second University of Naples,

Via L. De Crecchio, 7 - 80138 Naples, Italy

E-mail: amalia.forte@unina2.it

Phone: ++39-081-5665930

Fax $\quad++39-081-5667547$

Keywords: vascular injury; remodelling; microarrays; inflammation; blood flow; Renin-Angiotensin System.

Short title: Expression changes in contralateral carotids after injury

Figures: 4

Tables: 2

Words: 5392

Supplementary files: 5 


\section{ABSTRACT}

Vascular surgery aimed at stenosis removal induces local reactions often leading to restenosis. While extensive analysis has been focused on pathways activated in injured arteries, little attention has been devoted to associated systemic vascular reactions. Aim of this study was to analyse changes occurring in contralateral uninjured rat carotids in the acute phase following unilateral injury.

WKY rats were submitted to unilateral carotid arteriotomy. Contralateral uniunjured carotids were harvested from 4 hrs to 7 days after injury. Carotids were also harvested from sham-operated rats and from uninjured rats. Carotid morphology and morphometry were examined. Affymetrix microarrays were used for differential analysis of gene expression. A subset of data was validated by Real-Time RT-PCR and verified at protein level by Western Blot.

1011 genes were differentially regulated in contralateral uninjured carotids from 4 hrs to 7 days after arteriotomy $(p<0.0001$, fold-change $\geq 2)$ and were classified in 19 gene ontology functional categories. To a lesser extent, mRNA variations occurred also in carotids of sham-operated rats. Among the changes, up-regulation of members of the Renin-Angiotensin System was detected, with possible implications for vasocompensative mechanisms induced by arteriotomy. In particular, a selective increase of the N-domain Angiotensin Converting Enzyme 69-kDa isoform, and not the classical somatic $195 \mathrm{kDa}$ isoform, was highlighted in contralateral uninjured carotids, suggesting that this $69-\mathrm{kDa}$ isoenzyme could influence the local Angiotensin II production.

In conclusion, systemic reactions to injury occur in the vasculature, with potential clinical relevance and suggesting caution in the choice of control during experimental design in vivo. 


\section{INTRODUCTION}

Surgical procedures to correct atherosclerotic arterial narrowing are always associated with some degree of injury to the vessel wall, which carries the risk of reocclusion of the vessel because of neointima and remodeling stimulated by the injury. This remains a serious problem despite recent advances in the use of drug-eluting stents [1]. Accordingly, the restenosis problem has been addressed by several approaches, including large-scale transcriptome analysis, to characterize patterns in the vascular response to injury with the aim to identify possible targets for therapy.

Animal studies and clinical observations indicate that change in carotid blood flow subsequent to unilateral carotid stenosis and/or endarterectomy or angioplasty have effects on flow in contralateral carotids [2]. This serves to compensate for the disturbed blood flow, but may also affect the structure and function of the uninjured artery, which may have significant implications for the comparison of injured vs. contralateral uninjured arteries, as is often done. In addition, it is well known that vascular injury triggers the release in the plasma of a number of molecular mediators, including cytokines and growth factors, that can trigger a systemic pathophysiological effect which may be important for the clinical outcome. Observations in contralateral uninjured arteries following unilateral carotid surgery would be expected to elucidate such systemic effects. In previous studies [3] we set up a model of rat carotid arteriotomy that mimics the injury affecting the arteries of patients submitted to endarterectomy rather than the trauma induced by standard balloon angioplasty. Our investigations, together with other studies based on a deep vascular injury [4,5], support the key role of adventitia and of perivascular tissues in arterial stenosis. Recently, we reported time-resolved expression profiles from carotid arteries subjected to arteriotomy [6]. Here we have used microarrays to analyse the time-dependent transcriptome variations in contralateral uninjured rat carotids after arteriotomy. The results reveal that substantial changes in gene expression occur in these vessels and in shamcontrol carotids, thus implying a systemic reaction of the vasculature to surgery. 


\section{Methods}

\section{Animals}

Studies were carried out on 12 week-old inbred male WKY rats (300-310 g) (Charles Rivers, France). All animals were handled in compliance with the "Guide for the Care and Use of Laboratory Animals" published by the US National Institute of Health (NIH publication No. 85-23, revised 1996). All protocols were approved by the Animal Care and Use Committee of the Second University of Naples. Rats were acclimatized and quarantined for at least one week before undergoing surgery.

\section{Vascular Injury}

Arteriotomy of rat common carotid artery was performed as already published [7]. Briefly, a plastic Scanlom clamp for coronary artery grafting was placed for 10 seconds on the carotid causing a crushing lesion to the vessel. At the same point where the clamp was applied, a $0.5 \mathrm{~mm}$ longitudinal incision was done through the full thickness of the carotid. The incision did not cross to the other side of the vessel. Haemostasis was obtained with a single adventitial 8.0-gauge polypropylene stitch. Once bleeding stopped, the carotid artery was carefully examined and blood pulsation was checked distally to the incision.

Sham-control rats were submitted only to skin incision and common carotid exposure, without other manipulation or vagus nerve dissection. Carotids were harvested after $30 \mathrm{~min}$, corresponding to the average time taken for arteriotomy surgery.

\section{Histological analysis}

Carotid arteries were harvested at 4 hrs, 48 hrs, 7 days, 14 days, 21 days and 30 days after arteriotomy ( $\mathrm{n}=4$ for each group). The vessels were fixed in $4 \%$ buffered formaldehyde, dehydrated and embedded in paraffin. $5 \mu \mathrm{m}$ cross-sections were stained with hematoxylin-orcein for nucleus and elastic fiber staining, respectively. Image screening and photography of serial cross-sections were performed using a Leica IM 1000 System. Lumen and medial areas were measured using the Leica IM 1000 software. The lumen and medial areas of each injured carotid were normalized with respect to the ipsilateral distal region. Measurements were performed by two independent observers.

\section{RNA extraction/labeling}

Contralateral uninjured carotid arteries were harvested at $4 \mathrm{hrs}, 48 \mathrm{hrs}$ and 7 days after arteriotomy. Carotids from uninjured rats and from sham-control rats were also harvested $(n=3$ pools of 5 carotids each for all groups). Total RNA was extracted using the RNeasy mini kit (Qiagen). During extraction, RNA was treated with DNase (Qiagen) to remove DNA contamination. RNA 
concentration was measured using a NanoDrop ND-1000 spectrophotometer (NanoDrop Technologies). Total RNA integrity was verified by electrophoresis on denaturing 1\% agarose gel containing highly sensitive 2x GelStar Nucleic Acid Stain (Lonza) to ensure the absence of sample degradation. Absence of residual DNA was verified by PCR on total RNA without reverse transcription. $200 \mathrm{ng}$ of each RNA sample were amplified and biotin-labeled with two rounds of amplification following the Affymetrix small sample labeling protocol.

\section{Microarray hybridization and analysis:}

$15 \mu \mathrm{g}$ of each biotinylated cRNA were hybridized to Genechips 2302.0 (Affymetrix). Microarray hybridization and analysis were performed as previously published [6]. Results were filtered for flag (presence call), then for fold-change (FC) $\geq 2$, obtaining a total of 15600 probe sets differentially expressed in the different conditions.

Statistical analysis was performed by the two-way ANOVA using a parametric test with variances assumed equal, p-value cut-off 0.0001. The Bonferroni multiple testing correction was applied. The false discovery rate under these conditions was proximal to zero (Supplemental file 1). Final data comply with the Minimum Information About a Microarray Experiment (MIAME) requirements and have been loaded in the EMBL-European Bioinformatics Institute (EBI) public database with the accession number E-MEXP-1278 (www.ebi.ac.uk). Differentially expressed genes were grouped for similar biological processes according to gene ontology (GO) definitions (www.geneontology.org) (supplemental file 2). A gene was associated with a gene ontology term if it was annotated by this term or by its child. Hierarchical clustering was performed on the gene lists by the Gene Tree algorithm using as similarity measure the Pearson correlation and the clustering algorithm average linkage.

Significant biological pathways implicated in time-dependent carotid reaction to arteriotomy and gene product association networks have been identified through DAVID (Database for Annotation, Visualization and Integrated Discovery) at http://david.abcc.ncifcrf.gov and by BioRag (Bioresource for array genes) at http://www.biorag.org (Supplemental files 3, 4).

\section{Quantitative real-time $R T$-PCR}

Quantitative real-time PCR (MJ Opticon II, Bio-Rad) was used to determine the copy number of mRNA for 8 genes (c-myc, AT1, ACE, Clcf1, Agt, Id2, Mcp-1, IL-1 $\beta$ ) in contralateral uninjured carotids in comparison to carotids from uninjured rats. Real Time RT-PCR was performed as previously published [6]. Validation of the microarray data was considered to be a significant $(p<0.05)$ change in normalized PCR copy number in the same direction found in the microarray data. GAPDH was chosen as reference housekeeping gene. Relative quantitative RT-PCR was used to 
determine the fold difference for genes. The PCR efficiency was determined for each primer pair and was calculated using a dilution series and MJ Opticon II analysis software.

\section{Western blot}

Contralateral uninjured carotid arteries were harvested at $4 \mathrm{hrs}(\mathrm{n}=3), 48 \mathrm{hrs}(\mathrm{n}=3=$ and 7 days $(\mathrm{n}=4)$ after arteriotomy. Carotids from uninjured rats $(n=3)$ and from sham-control rats $(n=3)$ were also harvested. The vessels were immediately frozen in liquid nitrogen and then stored at $-80^{\circ} \mathrm{C}$. The frozen vessels were pulverized in liquid nitrogen and dissolved in Laemmli SDS-sample buffer. Protein concentrations were determined using EZQ Protein Quantitation assay (Molecular Probes).

Proteins were separated by SDS polyacrylamide gel electrophoresis using the Criterion precast gel system (Biorad). The same amount of protein $(20 \mu \mathrm{g})$ was loaded in each lane. The amounts of Arginase I and Angiotensin Converting Enzyme (ACE) were determined by Western blot (WB) using antibodies from BD Bioscience, Pharmingen (Arginase I) and Santa Cruz Biotechnology (ACE H170). Bands were visualized using enhanced chemiluminescence (Pierce) and analyzed with Quantity One software (Bio-Rad).

\section{Statistical analysis}

All statistical analysis was performed using GraphPad software (Prism 4.0). Statistical significance was determined using two-way ANOVA followed by Bonferroni's multiple comparison test.

Licenced copy. Copying is not permitted, except with prior 6 permission and as allowed by law. (C) 2008 The Authors Journal compilation (C) 2008 Biochemical Society 


\section{RESULTS}

\section{Histological analysis of arteriotomy-injured carotids and of contralateral uninjured carotids:}

Morphological analysis of cross-sections of carotids harvested from 4 hrs up to 30 days after arteriotomy showed changes mainly related to remodeling phenomena, while limited focal neointima was evident only in a few cases. Morphometric analysis revealed a progressive lumen reduction, with

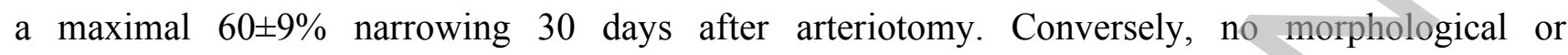
morphometric differences among contralateral uninjured carotids harvested at different time points after arteriotomy were evident (data not shown).

\section{Microarray transcriptome analysis in contralateral uninjured carotids at different time points after unilateral arteriotomy:}

The microarray data showed consistency in gene expression amongst the replicates within each experimental group, but evidence of substantial transcriptional differences in contralateral uninjured carotids at different time points after unilateral arteriotomy was found. In Supplementary file 1 are listed a total of $1011 \mathrm{mRNA}$ from uninjured carotids that were significantly affected at least at one time point after arteriotomy. Gene names, gene descriptions, biological function, Affymetrix Ids, accession numbers and expression levels are provided.

In uninjured carotids harvested at $4 \mathrm{hrs}, 48 \mathrm{hrs}$ and 7 days after arteriotomy, 728, 480 and 455 mRNAs, respectively, were significantly altered. Among the 1011 arteriotomy-modulated genes, the biological functions of 522 genes are known (Supplementary File 2). The remaining 489 genes were expressed sequence tags (ESTs), also listed in Supplementary file 2. In general, mRNA changes occurring in contralateral uninjured carotids after arteriotomy overlapped with changes detected in injured carotids relative to basal mRNA levels in uninjured rat carotids [6], but with a lower FC magnitude. In a few cases, mRNA expression trends showed an opposite behaviour in injured and contralateral uninjured carotids.

Heat map in Fig. 1 highlights, through a reference colour scale (green $=0$; red $=5$ ), the different temporal trends exhibited by GO functional categories. For example, genes included in cytoskeleton and contractile apparatus GO category clearly showed a biphasic up-regulation at $4 \mathrm{hrs}$ and 7 days after arteriotomy, while other groups of genes (e.g. nucleic acid metabolism and protein metabolism) exhibited a marked up-regulation only at $4 \mathrm{hrs}$ after arteriotomy and then gradually decreased to basal levels. Fig. 2 highlights the number of modulated genes for each GO functional category at different time points after arteriotomy in contralateral uninjured carotids. Genes included in each GO functional category are listed and described in Supplementary file 2.

Graphs in Fig. 3 highlight representative time-dependent differences between contralateral uninjured carotids and injured carotids. Graphs in Fig. 3A compare mean FC values for overall genechip data in 
injured carotids (data from ref. 6) and in contralateral uninjured carotids (present study). Panels B-E show similar data for the functional GO categories immune response, cytoskeleton and contractile apparatus, signal transduction and neurophysiological process, respectively. Graphs in Fig. 3F-L report the number of up-and down-regulated genes within the corresponding data sets. These graphs reveal a generally greater FC in arteriotomy-injured carotids, together with a greater number of activated genes and a more persistent activation in injured carotids in comparison with contralateral uninjured carotids. The graphs we selected for Fig. 3 reflect a general behavior observed for all the 19 GO functional categories we identified (Fig. 2).

Microarray data have been further analyzed to identify significant functional pathways activated after unilateral arteriotomy. A total of 15 most significant KEGG pathways $(p<0.1)$ have been identified by conjunct application of DAVID and BioRag (Table 1, Supplementary Fig. 3). A modified Fisher Exact $\mathrm{p}$-value $(0<\mathrm{p}<1)$ is provided for each functional KEGG pathway. The smaller the $\mathrm{p}$-value, the more over-represented (enriched) will this pathway be in the gene list, relative to random selection.

Supplementary file 4 includes the full list of genes included in each most significant pathway. The gene product association networks extracted from the 15 significant KEGG pathways are shown in Supplementary Fig. 4A and 4B. The core of the network in Supplementary Fig. 4A is constituted by genes involved in MAPK signaling (green line), focal adhesion (yellow line) and cytokine-cytokine receptor interaction (red line).

Of interest, we identified a subgroup of 21 mRNAs that were significantly modified exclusively in contralateral uninjured and not in arteriotomy-injured carotids (Supplementary file 5). 12 out of the 21 mRNAs had a well-described biological function in rat, and of these 7 were up-regulated and 5 down-regulated after arteriotomy. ACE was selectively up-regulated in uninjured contralateral carotids at $48 \mathrm{hrs}$ and 7 days after arteriotomy (FC 4.57 and 5.61, respectively). The marked upregulation of ACE in uninjured carotids could be responsible for an increase of local Angiotensin (Ang) II production. We also detected a marked 8.63-fold increase of Angiotensinogen (Agt) mRNA in contralateral carotids harvested 4 hrs after arteriotomy vs. a slight 2.12-fold increase measured in injured carotids [6]. Finally, the mRNA coding for the AT1 receptor for Ang II showed a comparable 3.39 and 4.37-fold increase in uninjured and injured carotids harvested 4 hrs after arteriotomy, respectively. Despite the subset of 21 genes selectively varied in contralateral uninjured carotids, we did not detect substantial differences in KEGG pathways identified in contralateral carotids in comparison to arteriotomy-injured carotids [6]. This is because the 12 selectively modified genes with described biological functions were found to belong to different functional pathways. 


\section{Real Time RT-PCR validation of microarray data:}

Real Time RT-PCR was done on a subset of mRNAs, either up- (c-myc, AT1, ACE, Agt, Mcp-1, IL$1 \beta)$ or down-regulated (Id2, Clcf1) after arteriotomy. These mRNAs were chosen as representative of pathophysiological processes of interest in our injury model (e,g. immune reaction, nucleic acid metabolism, vascular remodeling), displaying a FC ranging from a high magnitude to near the 2-fold cut-off. Gene expression data were normalized with respect to mRNAs from carotids of uninjured rats.

The trend of Real Time RT-PCR results overlapped with the microarray data, although the magnitude of the FC was not identical (Table 2). For some genes, the Real Time RT-PCR revealed additional significant changes that were not included among microarray data. This could be related to the greater sensitivity of Real Time PCR compared to microarrays, or to filtering out of some results during statistical analysis due to the standard deviation in microarray replicates.

\section{Western Blot verification of microarray data:}

We verified by WB the expression levels of ACE and Arginase I on lysates from contralateral uninjured carotids at $4 \mathrm{hrs}, 48 \mathrm{hrs}$ and 7 days after arteriotomy, as well as from sham-control carotids and from carotids of uninjured rats. Results were normalised with respect to GAPDH house keeping gene level.

N-domain ACE (69-kDa) showed a 2.24-fold increase in sham-control carotids and a 2.1- and 1.85fold increase in contralateral uninjured carotids $4 \mathrm{hrs}$ and $48 \mathrm{hrs}$ after arteriotomy. The expression of $\mathrm{N}$-domain ACE then turned back to basal levels 7 days after unilateral arteriotomy (Fig. 4A). We did not detect the 195-kDa somatic isoform of ACE in carotid lysates, which was however present in control rat kidney lysate, together with the N-domain 69- and 136-kDa ACE isoforms (data not shown). The expression trend of the protein partially overlapped with that of mRNA, showing a significant 4.54- and a 5.61-fold increase in contralateral carotids harvested $48 \mathrm{hrs}$ and 7 days after unilateral arteriotomy, respectively. In sham-control carotids and in contralateral uninjured carotids harvested 4 hrs after arteriotomy, ACE mRNA increased but showed a variability that caused this to be filtered out during statistical analysis.

WB analysis revealed a 2.53-fold increase of Arginase I in contralateral uninjured carotids harvested $4 \mathrm{hrs}$ after arteriotomy and a 4.28-fold increase in sham-control carotids (Fig. 4B). The expression of Arginase I was then back to basal levels at $48 \mathrm{hrs}$ and 7 days after arteriotomy. The expression trend of the protein partially overlapped with that of mRNA, showing a significant 2.73-fold increase 48 hrs after arteriotomy, and a significant 1.7-fold increase in sham control carotids (not shown in microarray results as we selected a $\mathrm{FC} \geq 2$ ). 


\section{Microarray transcriptome analysis of sham-control carotids:}

Only 149 mRNAs were significantly altered in sham-control carotids harvested at 30 min after surgery (Supplementary File 1). In this case, arteries were not manipulated and the vagus nerve not dissected. The majority of them were assigned to the inflammatory response and to the cytoskeleton and contractile apparatus (Supplementary file 2). Pathway analysis revealed a significant activation of fatty acid synthesis, ECM-receptor interaction and cytokine-cytokine receptor interaction in shamcontrol carotids $(\mathrm{p}<0.1)$.

Interestingly, a subset of mRNAs from sham-control carotids showed an expression trend opposite to that observed in injured carotids and/or in contralateral uninjured carotids. In particular, this was the case for mRNAs involved in inflammatory response (e.g. the macrophage inflammatory protein-1 alpha receptor, IL-6, the serine peptidase inhibitors Spin2c and Serpinb2, RT1-Ba, the chemokine ligand 9) and of other heterogeneous factors (e.g. c-fos, Cox6a2, Scn9a, Phosphoglycerate mutase 2). These differences in mRNA expression between sham-control carotids and contralateral uninjured carotids may be related to feed-back regulative mechanisms and to the fact that sham-control carotids were harvested at $30 \mathrm{~min}$ after skin injury and carotid exposure, while contralateral carotids were harvested not earlier than 4 hrs after arteriotomy.

Licenced copy. Copying is not permitted, except with prior permission and as allowed by law. (C) 2008 The Authors Journal compilation (C) 2008 Biochemical Society 


\section{DISCUSSION}

The analysis of systemic inflammatory and compensatory effects triggered by vascular injury procedures can have clinical relevance and be helpful for a better comprehension of the pathophysiological events occurring during and after surgery.

High-density microarrays identified a large number of mRNA variations in contralateral uninjured carotids and sham-control carotids in a model of rat arteriotomy, allowing the dissection of patterns that could not have been discovered through analysis of individual genes. Microarrays were used in association with a method developed for mRNA linear amplification [8] that allowed the analysis of low amounts of RNA without affecting the fidelity of detection of differential gene expression and the false positive rate [9]. Sample pooling before RNA extraction was necessary because of the low yield of RNA obtained from single samples, as we extracted RNA only from small carotid segments corresponding to those submitted to arteriotomy (less then $2 \mathrm{~mm}$ in length). Carotid pooling allowed the recovery of a sufficient amount of RNA for check of sample integrity and DNA absence, microarray hybridisation and Real Time RT-PCR for data validation. We used a large base of biological samples ( $\mathrm{n}=15$ rats for each time point) and stringent parameters to filter the data to avoid false gene identification and assign a level of significance to the overall results.

Histological analysis did not reveal any significant morphological or morphometric change in contralateral uninjured carotids harvested at different time points after unilateral arteriotomy. This is in agreement with findings recently reported in a model of mouse carotid artery ligation, in which authors did not detect any proliferative activity in contralateral unligated vessels, but a prominently increased expression of Delta-like 1 gene [10].

Among the most significant KEGG pathways activated in contralateral uninjured carotids are those related to inflammation (e.g. antigen processing and presentation, B cell receptor signalling pathway, cytokine-cytokine receptor interaction), to cell-ECM adhesion and to the MAPK signalling pathway (Supplementary Fig. 3A). Of note, these pathways are not substantially different from those identified in arteriotomy-injured carotids [6].

Comparative analysis of GO categories in injured and contralateral uninjured carotids shown in Fig. 3 highlights differences in relative magnitude of FC and timing of variations of gene expression. In particular, the overall results show a lower mean FC value at all time points after arteriotomy in contralateral uninjured carotids than in injured carotids (Fig. 3A and F), together with differences in the number of regulated genes, most evident at 7 days after injury, when the majority of transcriptional activity in the contralateral artery, but not in injured carotids, has settled down. This indicates that variations of gene expression in contralateral uninjured carotids are transient. The overall results shown in Fig. 3A and F are similar to those obtained in the single GO categories (Fig. 
3B-E), with the exception of the GO category "cytoskeleton and contractile apparatus", (Fig. 3D), in which the mean $\mathrm{FC}$ value rises again at 7 days after arteriotomy in contralateral uninjured carotids.

The genes and the pathways activated in contralateral uninjured carotids are presumably strictly related to changes occurring in arteriotomy-injured carotids [6]. Cytokines secreted by arteriotomyinjured vessels and by perivascular tissues may produce systemic effects detectable at molecular level at distal sites, as in our study, and could be predictable of future events of clinical relevance. In this context, Raman et al. [11] showed that contralateral uninjured carotids of a cohort of patients submitted to unilateral endarterectomy had an annual rate of stenosis progression double that of ipsilateral carotids after endarterectomy. These authors did not identify any clinical or demographic risk factors associated with contralateral disease progression.

Other studies demonstrated that vascular injury causes changes in tissues of normal appearance located at a distance from the injury site. In particular, it has been demonstrated that SMC proliferation occurs at sites distant from vascular injury in aorta [12]. Moreover, patients with acute myocardial infarction showed a severe vasodilator abnormality involving not only resistance vessels in infarcted myocardium, but also those in myocardium perfused by normal coronary vessels [13]. Finally, it has been demonstrated that renal artery manipulation causes a centripetal spread of endothelial cell replication along the periureteric collateral artery [14]. The mediators of the above mentioned distal effects could be humoral vasoactive factors released immediately after the injury, able to play autocrine, paracrine and systemic effects. We have previously demonstrated that carotid arteriotomy induces synthesis of a number of mRNAs coding for cytokines, chemokines, hormones and growth factors, including IL-6 and Monocyte chemotactic protein (MCP-1/Ccl2) [6]. These two factors appear of particular interest for their effect on contralateral uninjured carotids, as they could play a regulative role through a feedback mechanism. In fact, IL-6 mRNA showed a 135-fold increase in rat carotids 4 hrs after arteriotomy [6] but a parallel marked decrease in contralateral uninjured and in sham-control carotids. These opposite expression trends could be related to a negative feedback mechanism of regulation of IL-6 mRNA in the cytokine cascade to inhibit excessive acute inflammation [15]. Conversely, the increase of MCP-1 mRNA both in injured and in contralateral carotids was presumably due to a positive loop induced by circulating MCP-1 released at the arteriotomy site, as suggested by data obtained in human CD14-positive monocytes [16].

The activation of mRNAs involved in the inflammatory reaction in contralateral uninjured carotids could be presumably related not only to the release of systemic mediators from the injury site, but also to altered blood flow, that has been recently demonstrated to be able to trigger an inflammatory response in mouse mesenteric arteries [17].

Finally, it can be also hypothesized that the vascular injury induced a marked inflammatory reaction triggering the priming of circulating leukocytes, which in turn can act as proatherogenic agents at 
local inflammatory sites. Similar mechanisms have been hypothesized in different vascular diseases $[18,19]$.

Among other pathways and processes activated in contralateral uninjured carotids, we detected the activation of a large number of genes involved in neurophysiological processes (e.g. Dbh, Nefl, Pcp4, Nef3, Syt1). In particular, Dbh and Nef3 mRNAs at 4 hrs after arteriotomy had increased much more than in injured carotids [6], probably as a part of a neurocompensatory reaction to vascular injury. Concomitantly, there was a massive decrease of the expression of genes involved in muscle contraction (e.g. troponin T2, myosin heavy chain polypeptides 3 and 8). In agreement with our data, Milner et al. [20] detected neurocompensatory responses to balloon injury in rat carotids, comprising damage to the perivascular nerves and a transient increase in the density of sensory neuropeptidecontaining nerves innervating the contralateral, uninjured side.

Microarray data indicated also an up-regulation of mRNAs involved in oxidative stress, in particular of NAD(P)H oxidase subunits 22phox, gp91phox and Ncf4 48 hrs and 7 days after arteriotomy. Moreover, we measured a 4-fold increase of the small GTPase Rac2 in contralateral uninjured carotids 7 days after arteriotomy. Sod2, which exerts an antioxidant activity, showed a transient slight increase $4 \mathrm{hrs}$ and $48 \mathrm{hrs}$ after arteriotomy, followed by a marked decrease 7 days after injury.

In an attempt to distinguish between variations of gene expression specific for contralateral carotids and early systemic reactions of the vasculature to surgical procedures, we analysed gene expression variations also in sham-control carotids harvested 30' after skin incision and carotid exposure. The mRNA variations in sham-control carotids indicated that the carotid exposure, even in the absence of carotid manipulation and vagus nerve dissection, was sufficient to trigger a rapid reaction in the vasculature, both at mRNA and protein level (Fig. 4; Supplementary file 1). The two major GO categories activated in sham-control carotids signalled an early systemic inflammatory response and activation of the contractile apparatus. The slight changes of mRNAs we observed in sham-operated controls are in contrast with some studies [20,21], but are in agreement with others [22,23].

Among the most significant KEGG pathways activated in contralateral uninjured carotids is the arginine and proline metabolism (Table 1). Arginase I plays a main role in this pathway, as it acts as a nitric oxide-synthase-alternative pathway for L-Arginine breakdown, leading to biosynthesis of urea and L-ornithine. Arginase I plays a role in the regulation of vascular tone and in vascular dysfunctions and is expressed by numerous cell types; moreover, Arginase I activity is increased by inflammatory molecules [24]. The increase of Arginase I mRNA and protein in contralateral uninjured and in sham-control carotids overlaps with the increase of Arginase I in arteriotomyinjured carotids [6]. The greater increase of Arginase I protein in sham-control carotids harvested 30 min after surgery (Fig. 4B) than in contralateral carotids at 4 hrs may indicate that this protein is 
increased rapidly by a systemic inflammatory reaction, as suggested by Wei et al. [24]. This could also involve a prompt regulation of vascular tone after injury.

Our microarray and Real Time RT-PCR data highlight a preferential up-regulation of mRNAs coding for elements of local Renin-Angiotenin System (RAS) (Agt, AT1 receptor, ACE) in contralateral uninjured carotids relative to arteriotomy-injured carotids [6]. In particular, ACE mRNA showed a marked increase in contralateral uninjured carotids, while it was substantially unchanged in arteriotomy-injured carotids [6]. The broad spectrum of substrates for ACE and its wide distribution indicates that this enzyme may be involved not only in cardiovascular homeostasis but also in pathophysiological processes. WB integrated these data revealing an increase of the N-domain ACE isoform $(69-\mathrm{kDa})$ in rat carotids and not of the classical somatic $195 \mathrm{kDa}$ ACE isoform (Fig. 4A). The N-domain 69-kDa and the 136-kDa N-domain ACE isoforms have been described in a variety of tissues, including aorta and heart [25], suggesting that these isoenzymes could influence local Ang II production, contributing to organ-specific regulation. In particular, the N-domain ACE isoforms are involved in the inactivation of vasodilator Ang1-7, a specific substrate for these isoforms, in addition to the conversion of Ang I to Ang II.

Despite the parallel increase of mRNAs, we cannot exclude that the Arginase I and ACE proteins detected in contralateral carotids derived from plasma residues infiltrating the intact vascular wall with a temporal expression profile related to changes in permeability of the vasculature. Nevertheless, we detected only the 69-kDa N-domain ACE isoform in carotids, distinct from the ACE soluble isoform released in the plasma, with a molecular size similar to that of human $195-\mathrm{kDa}$ somatic membrane-bound ACE [26].

The importance of local vascular RAS is well established. Its activation in contralateral carotids after arteriotomy is in agreement with other studies hypothesizing that a RAS-mediated compensatory response may be caused by an increase in Ang II receptor density [21] and demonstrating that chemical sympathectomy in rats submitted to angioplasty induced an increase of DNA synthesis mediated by Ang II receptors exclusively in contralateral carotids [27].

\section{CONCLUSIONS AND PERSPECTIVES}

The main findings of this study are that: i) carotid arteriotomy induces an extensive change of transcriptome in contralateral uninjured carotids; ii) local RAS is specifically up-regulated in contralateral uninjured carotids and in sham-control carotids, suggesting a role in the prompt regulation of vascular tone; iii) slight changes at mRNA and protein level have been detected also in sham control-carotids, indicating early systemic effects unrelated to altered carotid blood flow following arteriotomy; iv) contralateral uninjured carotids should be used with caution as reference control in vivo, since considerable changes occur also in these vessels. 
The absence of evident morphological variations in contralateral uninjured arteries despite variations at mRNA and protein levels was probably related to their intact vascular wall, which inhibited cell proliferation and remodelling phenomena.

This study used healthy WKY rats as experimental animal model. Rodent models have the limitation that atherosclerosis-related pathological changes do not occur unless the animals are given special diets and/or have genetic dyslipidemias, as in several genetically modified mouse strains. The current analysis of gene expression following injury to healthy vessels thus represents changes related to the injury by itself without the complication of dyslipidemia or plaque formation. An analysis of differential expression in a model of stenosis induced in hypercholesterolemic animals would be of interest to simulate processes taking place in human atherosclerotic arteries. The vascular wall affected by an atherosclerotic plaque can show basal inflammation, endothelial dysfunction and proliferative activity that may influence not only its reactions to injury, but also the reactions of contralateral uninjured carotids exposed to the same blood and tissue environment.

To our knowledge, this is the first microarray-based analysis of time-dependent gene expression profiling in contralateral uninjured arteries in a model of vascular injury. The results raise questions with regard to potential adverse systemic effects of vascular injury, of possible pathophysiological and clinical significance. 


\section{Acknowledgements}

To the memory of Prof. Antonino Cascino, deceased 6 April 2008.

This work has been partially supported by Swedish Research Council (71X-28), The Swedish Heart Lung Foundation, the Torsten and Ragnar Söderberg Foundations and the Vascular Wall Programme, Lund University, to P.H. and by Ministero della Sanità, PF Sanità 2003 "Patologie infettive e insulto chirurgico: studi di genomica e proteomica nel remodeling vascolare" to A.C.

We are grateful to Ms. Maria Rosaria Cipollaro for technical assistance.

Conflict of interest: none declared 


\section{LEGENDS}

Fig. 1: Time-dependent expression of genes clustered in functional groups. Each row corresponds to a single gene; each column corresponds to the mean of three different experiments (reference colour scale: green $=0 ;$ red $=5$ ).

Fig. 2: Functional GO classification of genes up-regulated (A) and down-regulated (B) at 4 hrs, 48 hrs and 7 days after arteriotomy in contralateral uninjured carotids.

Fig. 3: Mean fold-change (FG) values (A-E) and number of up- and down-regulated genes (L-H) at different time points after arteriotomy in injured and contralateral uninjured carotids for overall genechip results $(\mathrm{A}, \mathrm{L})$ and for the GO categories signal transduction, cytoskeleton and contractile apparatus, neurophysiological process and immune response (B-E, G-H). Data for injured carotids are from Ref. 6 and are shown here for comparison.

Fig. 4: Western Blot analysis of ACE and Arginase I expression in carotids from uninjured rats, sham-control rats and in contralateral uninjured carotids harvested at $4 \mathrm{hrs}, 48 \mathrm{hrs}$ and 7 days after arteriotomy. Original blots (A) and summarized data normalised with respect to GAPDH housekeeping gene $(B)$ (mean values, $n=3$ ) are shown.

Licenced copy. Copying is not permitted, except with prior permission and as allowed by law. (C) 2008 The Authors Journal compilation (C) 2008 Biochemical Society 


\section{Legends of supplementary files:}

Supplementary file 1: Differentially expressed mRNAs in contralateral uninjured rat carotids harvested at $4 \mathrm{hrs}, 48 \mathrm{hrs}$ and 7 days after arteriotomy and in sham-control carotids harvested 30' after injury (these data are also available at www.ebi.ac.uk).

Supplementary file 2: Microarray data classification according to their GO biological functions.

Supplementary file 3: Networks among genes included in significant (A) cellular and regulatory process and (B) metabolic KEGG pathways activated in contralateral uninjured carotids at $4 \mathrm{hrs}, 48$ hrs and 7 days after arteriotomy. The core of the network in A is constituted by genes involved in MAPK signalling (green line), cytokine-cytokine receptor interaction (red line) and focal adhesion (yellow line). The most significant pathway in B is arginine and proline metabolism. Faded nodes belong to pathways with a $\mathrm{p}>0.1$. The thickness of the edge is proportional to the number of pathways to which are associated two genes.

Supplementary file 4: Gene classification in KEGG pathways.

Supplementary file 5: mRNAs modified exclusively in contralateral uninjured carotids. 


\section{REFERENCES}

1) Camenzind, E. (2006) Treatment of in-stent restenosis--back to the future? N. Engl. J. Med. 355, 2149-2151.

2) van Everdingen, K.J., Klijn, C.J., Kappelle, L.J., Mali, W.P., van der Grond, J. (1997) MRA flow quantification in patients with a symptomatic internal carotid artery occlusion. The Dutch EC-IC Bypass Study Group. Stroke 28, 1595-1600.

3) Forte, A., Esposito, S., De Feo, M., et al. (2003) Stenosis progression after surgical injury in Milan hypertensive rat carotid arteries. Cardiovasc. Res. 60, 654-663.

4) Andersen, H.R., Maeng, M., Thorwest, M., Falk, E. (1996) Remodeling rather than neointimal formation explains luminal narrowing after deep vessel wall injury: insights from a porcine coronary (re)stenosis model. Circulation 93, 1716-1724.

5) Okamoto, E., Couse, T., De Leon, H., et al. (2001) Perivascular inflammation after balloon angioplasty of porcine coronary arteries. Circulation 104, 2228-2235.

6) Forte, A., Finicelli, M., De Luca, P., et al. (2007) Expression profiles in surgically-induced carotid stenosis: a combined transcriptomic and proteomic investigation. J. Cell. Mol. Med. in press.

7) Forte, A., Galderisi, U., De Feo, M, et al. (2005) c-Myc antisense oligonucleotides preserve smooth muscle differentiation and reduce negative remodelling following rat carotid arteriotomy. $\mathrm{J}$ Vasc. Res. 42, 214-225.

8) Eberwine, J. (1996) Amplification of mRNA populations using aRNA generated from immobilized oligo(dT)-T7 primed cDNA. Biotechniques 20, 584-591.

9) Patel, O.V., Suchyta, S.P., Sipkovsky, S.S., et al. (2005) Validation and application of a high fidelity mRNA linear amplification procedure for profiling gene expression. Vet. Immunol. Immunopathol. 105, 331-342. 
10) Miceli-Libby, L., Johnson, M.J., Harrington, A., Hara-Kaonga, B., Ng, A.K., Liaw, L. (2008) Widespread delta-like-1 expression in normal adult mouse tissue and injured endothelium is reflected by expression of the Dll1LacZ locus. J. Vasc. Res. 45, 1-9.

11) Raman, K.G., Layne, S., Makaroun, M.S., et al. (2004) Disease progression in contralateral carotid artery is common after endarterectomy. J. Vasc. Surg. 39, 52-57.

12) Reidy, M.A. (1990) Proliferation of smooth muscle cells at sites distant from vascular injury. Arteriosclerosis 10, 298-305.

13) Uren, N.G., Crake, T., Lefroy, D.C., de Silva, R., Davies, G.J., Maseri A. (1994) Reduced coronary vasodilator function in infarcted and normal myocardium after myocardial infarction. N. Engl. J. Med. 331, 222-227.

14) Hollenberg, N.K., Odori, T. (1987) Centripetal spread of arterial collateral endothelial cell hyperplasia after renal artery stenosis in the rat. Circ. Res. 60,398-401.

15) Saito, H., Patterson, C., Hu, Z., et al. (2000) Expression and self-regulatory function of cardiac interleukin-6 during endotoxemia. Am. J. Physiol. Heart Circ. Physiol. 279, H2241-2248.

16) Sakai, N., Wada, T., Furuichi K., et al. (2006) MCP-1/CCR2-dependent loop for fibrogenesis in human peripheral CD14-positive monocytes. J. Leukoc. Biol. 79, 555-563.

17) Bakker, E.N., Matlung, H.L., Bonta, P., de Vries, C.J., van Rooijen, N., Vanbavel, E. (2008) Blood flow-dependent arterial remodeling is facilitated by inflammation but directed by vascular tone. Cardiovasc. Res. in press.

18) Kolb, F., Heudes, D., Mandet, C., et al. (1996) Presensitization accelerates allograft arteriosclerosis. Transplantation 62, 1401-1410.

19) Jacobi, J., Sela, S., Cohen, H.I., Chezar, J., Kristal, B. (2006) Priming of polymorphonuclear leukocytes: a culprit in the initiation of endothelial cell injury. Am. J. Physiol. Heart Circ. Physiol. 290, H2051-2058. 
20) Milner, P., Crowe, R., Loesch, A., Anglin, S., Burnstock, G., McEwan, J.R. (1997) Neurocompensatory responses to balloon-catheter-induced injury of the rat carotid artery. J. Vasc. Res. 34, 31-40.

21) Fukada, S.Y., Correa, F.M., Ramalho, L.N., Mizusaki, C.I., de Oliveira, A.M. (2004) Perivascular injury leads to a reduction in vascular reactivity of the collared and to an enhancement on contralateral carotid artery of rats. Cardiovasc. Pathol. 13, 251-259.

22) Connolly, E., Bouchier-Hayes, D.J., Kaye, E., Leahy, A., Fitzgerald, D., Belton, O. (2002) Cyclooxygenase isozyme expression and intimal hyperplasia in a rat model of balloon angioplasty. $\mathrm{J}$. Pharmacol. Exp. Ther. 300, 393-398.

23) Li, J.M., Zhang, X., Nelson, P.R., et al. (2007) Temporal evolution of gene expression in rat carotid artery following balloon angioplasty. J. Cell. Biochem. 101, 399-410.

24) Wei, L.H., Jacobs, A.T., Morris, S.M., Jr., Ignarro, L.J. (2000) IL-4 and IL-13 upregulate arginase I expression by cAMP and JAK/STAT6 pathways in vascular smooth muscle cells. Am. J. Physiol. Cell. Physiol. 279, C248-256.

25) Ronchi, F.A., Andrade, M.C., Carmona, A.K., Krieger, J.E., Casarini, D.E. (2005) N-domain angiotensin-converting enzyme isoform expression in tissues of Wistar and spontaneously hypertensive rats. J. Hypertens. 23, 1869-1878.

26) Baudin, B., Tahraoui, A., Baumann, F.C., Robic, D., Drouet, L., Legrand, Y. (1991) Purification and analysis of lung and plasma angiotensin I-converting enzyme by high-performance liquid chromatography. Protein Expr. Purif. 2, 412-419.

27) Bruijns, R.H., van Kleef, E.M., Smits, J.F., De Mey, J.G., Daemen, M.J. (1998) Effects of chemical sympathectomy on angiotensin II-induced neointimal growth in the balloon-injured rat carotid artery. J. Vasc. Res. 35, 124-133. 


\begin{tabular}{|c|c|c|c|c|c|c|}
\hline & & ber o & & & gnifican & \\
\hline KEGG Pathways & Time & after & iotomy & Time & s after: & otomy \\
\hline $\begin{array}{l}\text { Cellular and regulatory } \\
\text { pathways }\end{array}$ & $4 h$ & $48 \mathrm{~h}$ & 7 days & $4 h$ & $48 \mathrm{~h}$ & 7 days \\
\hline Focal adhesion & 21 & 16 & 12 & $9.2 \mathrm{E}-4$ & $3.8 \mathrm{E}-3$ & $1.4 \mathrm{E}-2$ \\
\hline ECM-receptor interaction & 10 & & 6 & 7.1E-3 & & 4.2E-2 \\
\hline $\begin{array}{l}\text { Antigen processing and } \\
\text { presentation }\end{array}$ & 9 & 8 & & $6.1 \mathrm{E}-2$ & $3.8 \mathrm{E}-2$ & \\
\hline Cell adhesion molecules (CAMS) & 14 & 10 & & $3.1 \mathrm{E}-2$ & $9.7 \mathrm{E}$ & \\
\hline Hematopoietic cell lineage & & 8 & 6 & & & 4.4E-2 \\
\hline B cell receptor signalling pathway & 8 & & 6 & $4.0 \mathrm{E}-2$ & & $3.1 \mathrm{E}-2$ \\
\hline $\begin{array}{l}\text { Cytokine-cytokine receptor } \\
\text { interaction }\end{array}$ & 12 & 12 & & $9.0 \mathrm{E}-2$ & & \\
\hline $\begin{array}{l}\text { Toll-like receptors signaling } \\
\text { pathway }\end{array}$ & & 7 & 6 & & & $3.7 \mathrm{E}-2$ \\
\hline MAPK signaling pathway & 18 & & & 4.41 & & \\
\hline $\begin{array}{l}\text { Complement and coagulation } \\
\text { cascades }\end{array}$ & & & 5 & & & $5.7 \mathrm{E}-2$ \\
\hline Cell communication & 10 & & & & & \\
\hline Type I diabetes mellitus & & 7 & & & $3.6 \mathrm{E}-2$ & \\
\hline Tgf-beta signaling pathway & 8 & & & & & \\
\hline Axon guidance & & & 2 & & & $5.7 \mathrm{E}-2$ \\
\hline Metabolic pathways & & & & & & \\
\hline Arginine and proline metabolism & & & 4 & & & 7.8E-2 \\
\hline
\end{tabular}

Table 1: Most significant KEGG pathways identified by DAVID software in contralateral uninjured carotids harvested at different time points after arteriotomy $(\mathrm{p}<0.1)$. The number of genes included in each pathway at different points after arteriotomy and the $\mathrm{P}$ values are reported. The p-value provided for each functional KEGG pathway is a modified Fisher Exact p-value. The smaller is the pvalue, the more enriched will be the pathway and consequently a gene list will be specifically associated (enriched) in a certain pathway than random chance. 


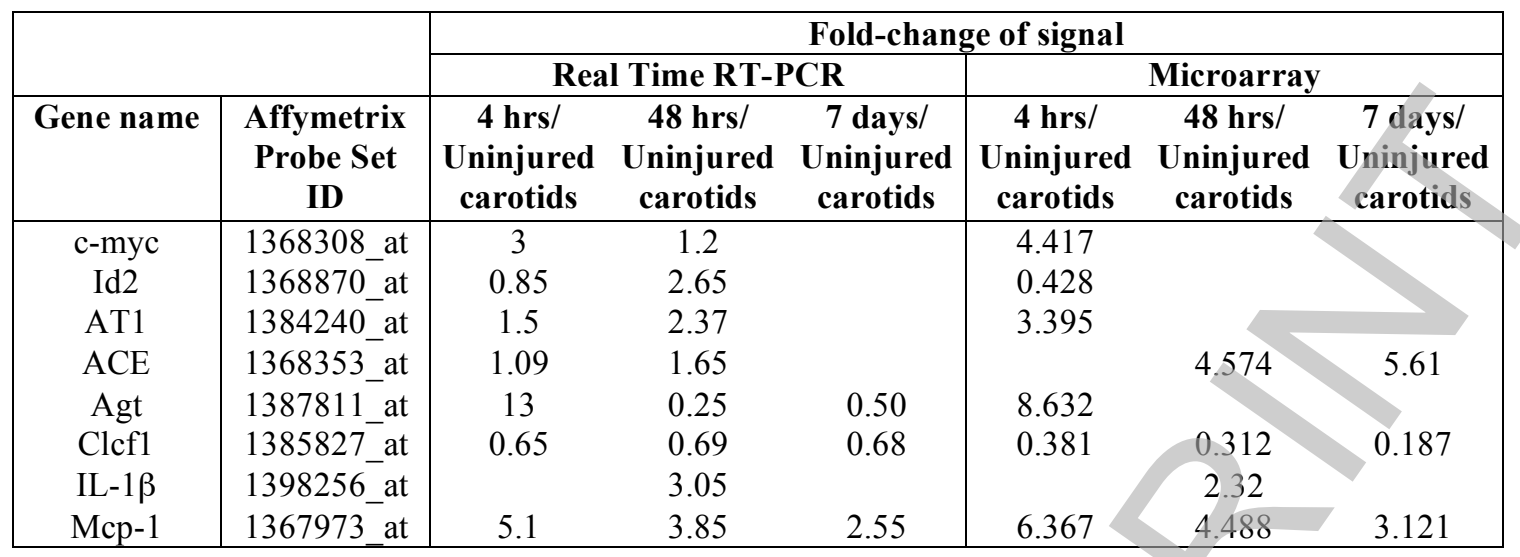

Table 2: Relative changes of gene expression as assessed by Real Time RT-PCR in contralateral uninjured carotids harvested $4 \mathrm{hrs}, 48 \mathrm{hrs}$ and 7 days after arteriotomy and comparison with related microarray data ( $n=3$ RNA pools for each time point). Only statistically significant variations $(p<0.05)$ of gene expression in comparison to carotids from uninjured rats were reported.

Licenced copy. Copying is not permitted, except with prior 23 ermission and as allowed by law. (C) 2008 The Authors Journal compilation (C) 2008 Biochemical Society 

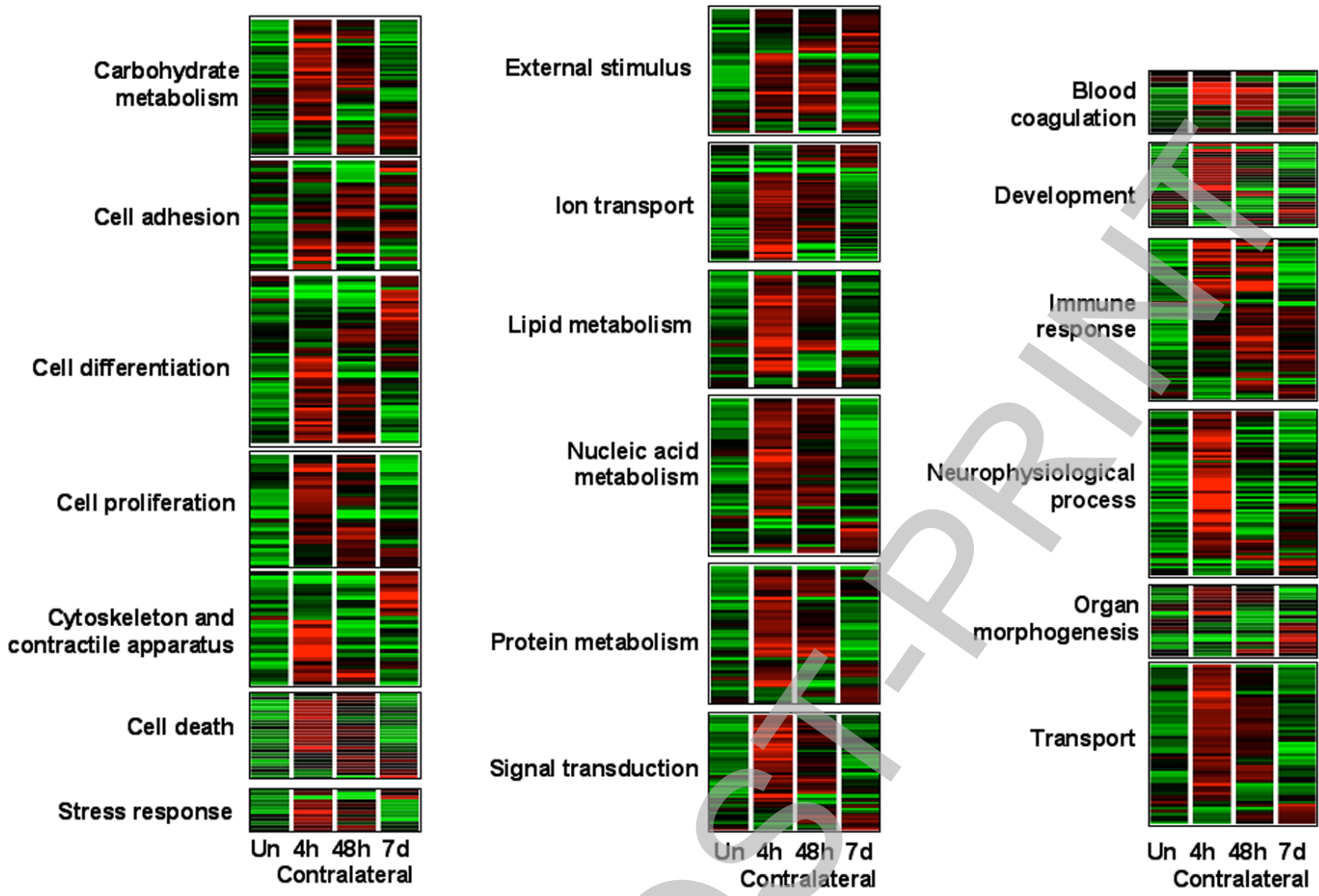

Figure 1

Licenced copy. Copying is not permitted, except with prior 24 permission and as allowed by law. (C) 2008 The Authors Journal compilation (C) 2008 Biochemical Society 


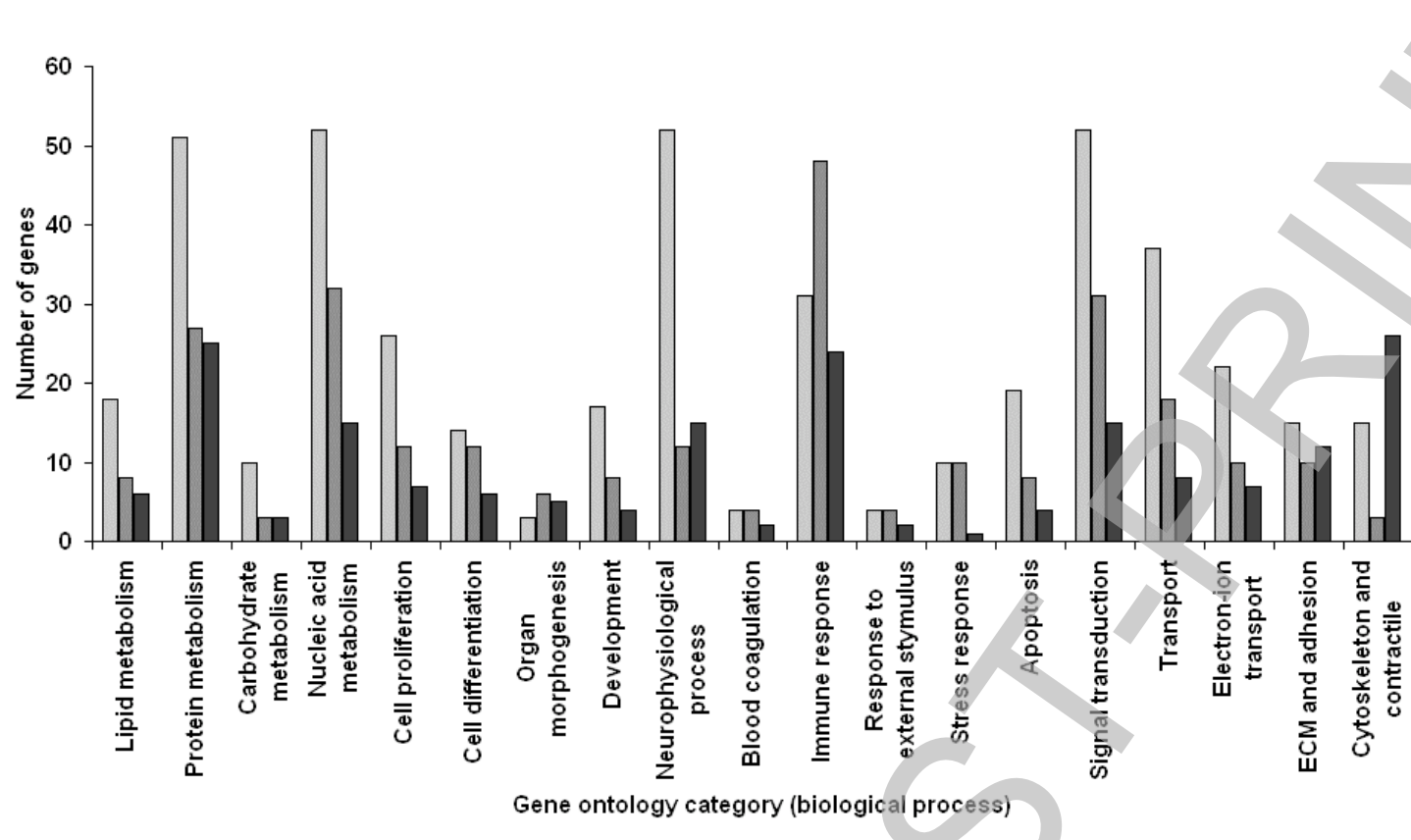

Gene ontology category (biological process)

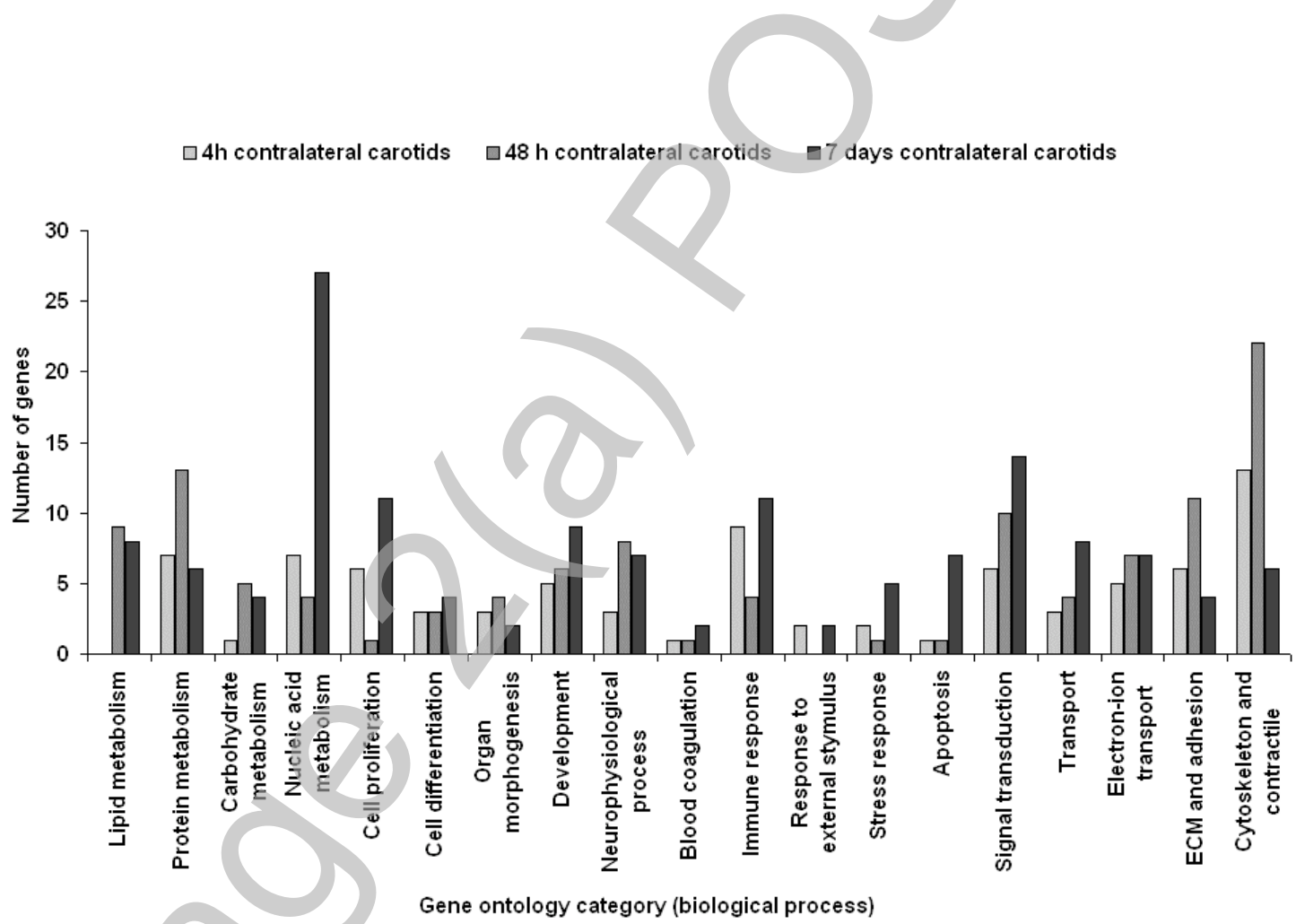

\section{Figure 2}

Licenced copy. Copying is not permitted, except with prior 25 permission and as allowed by law. (C) 2008 The Authors Journal compilation (C) 2008 Biochemical Society 
A

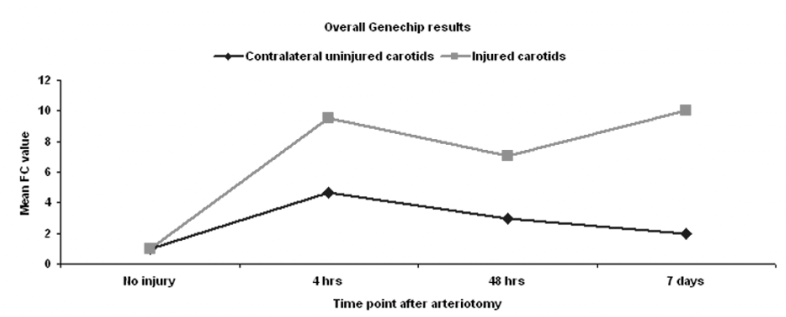

B

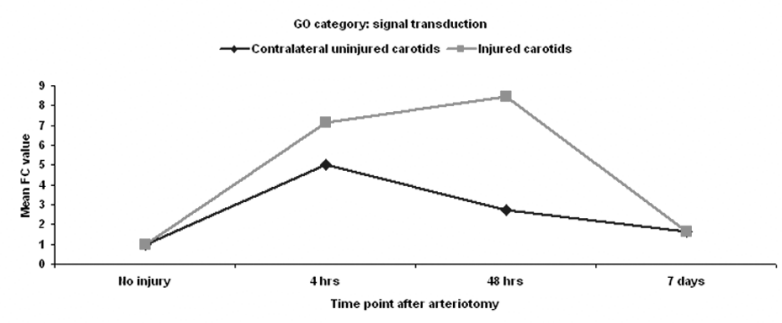

C

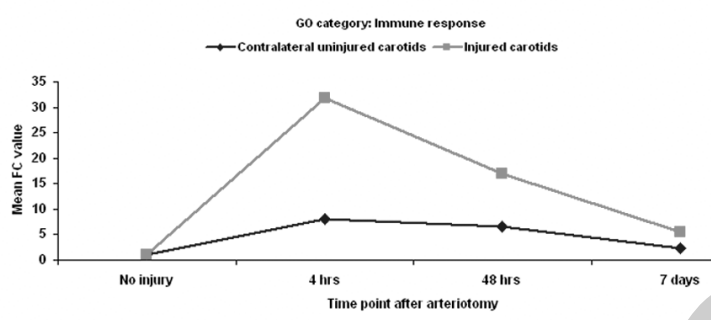

D

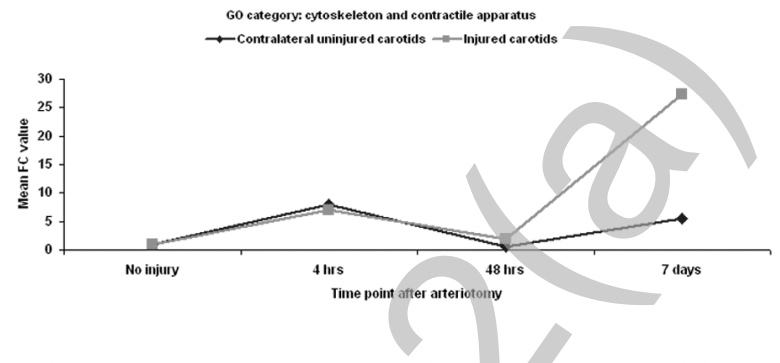

E

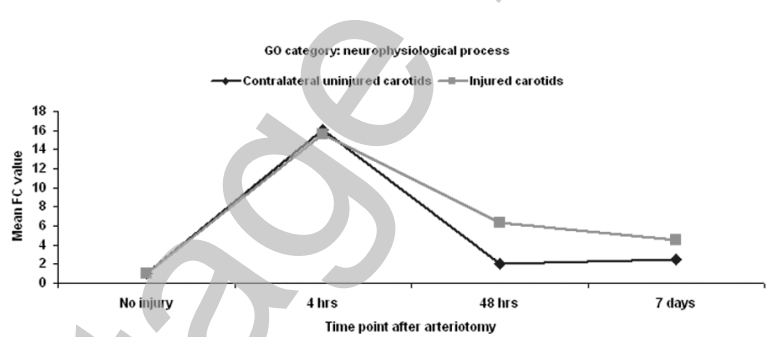

F

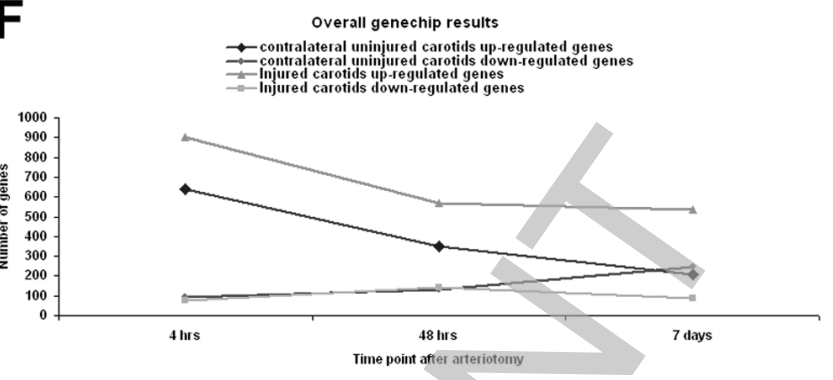

G
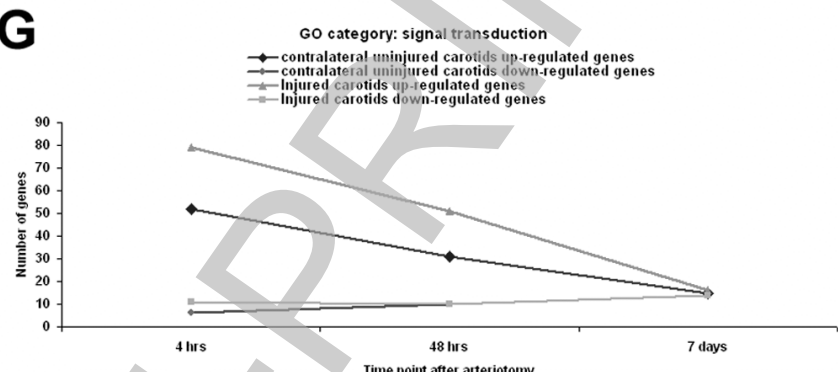

H GO category: immune response $\Rightarrow$ - contralateral uninumed carotids yp.regulated genes

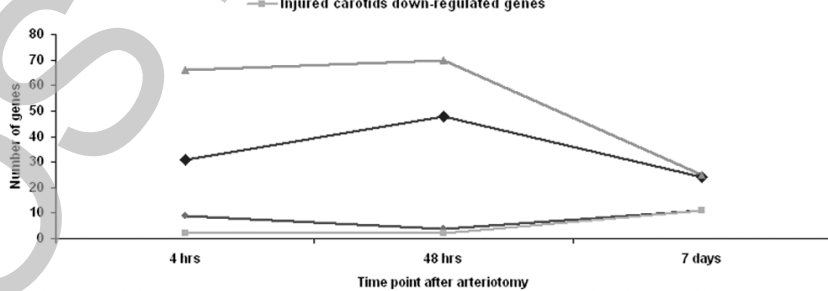

I

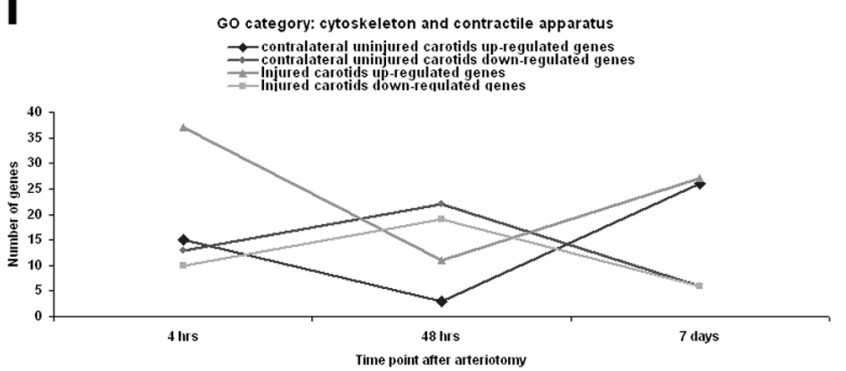

L GO cate gory: neurophysiological process

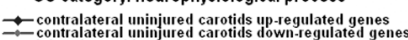

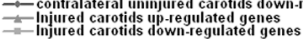

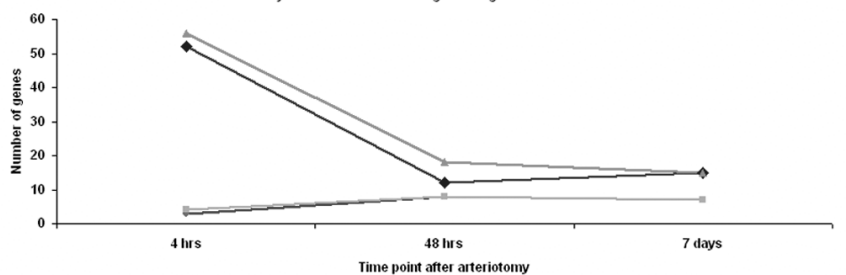

\section{Figure 3}

Licenced copy. Copying is not permitted, except with prior permission and as allowed by law. (C) 2008 The Authors Journal compilation (C) 2008 Biochemical Society 
A

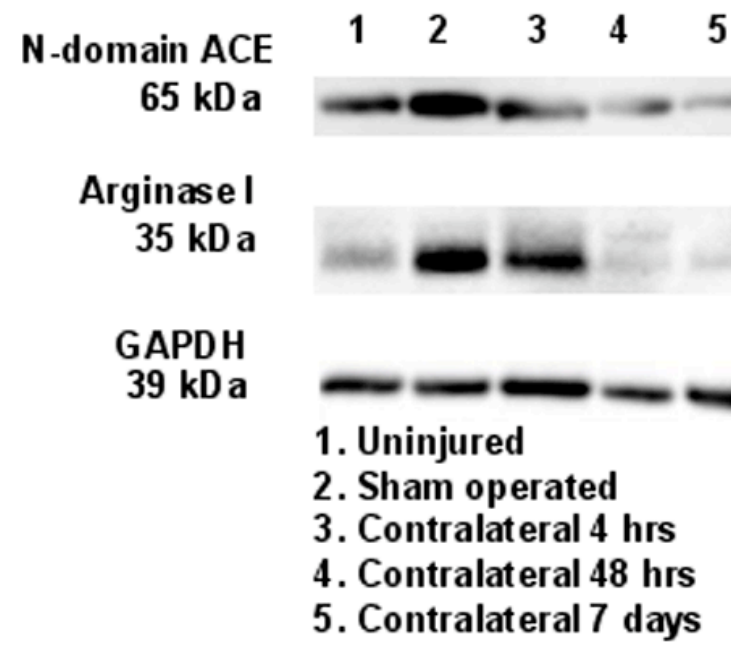

B

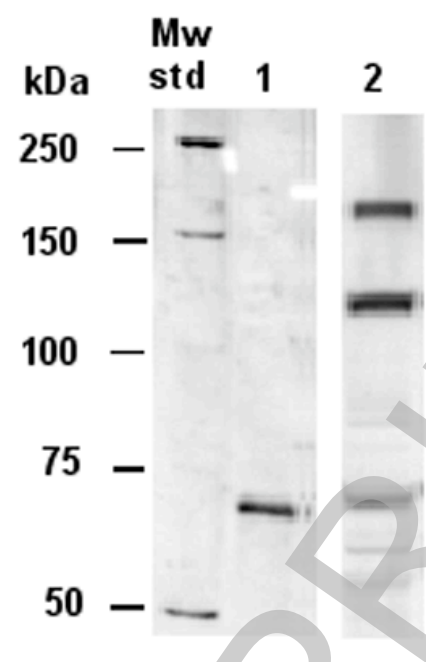

1. ACE (contralateral carotid)

2. ACE (mouse kidney)

C

Figure 4

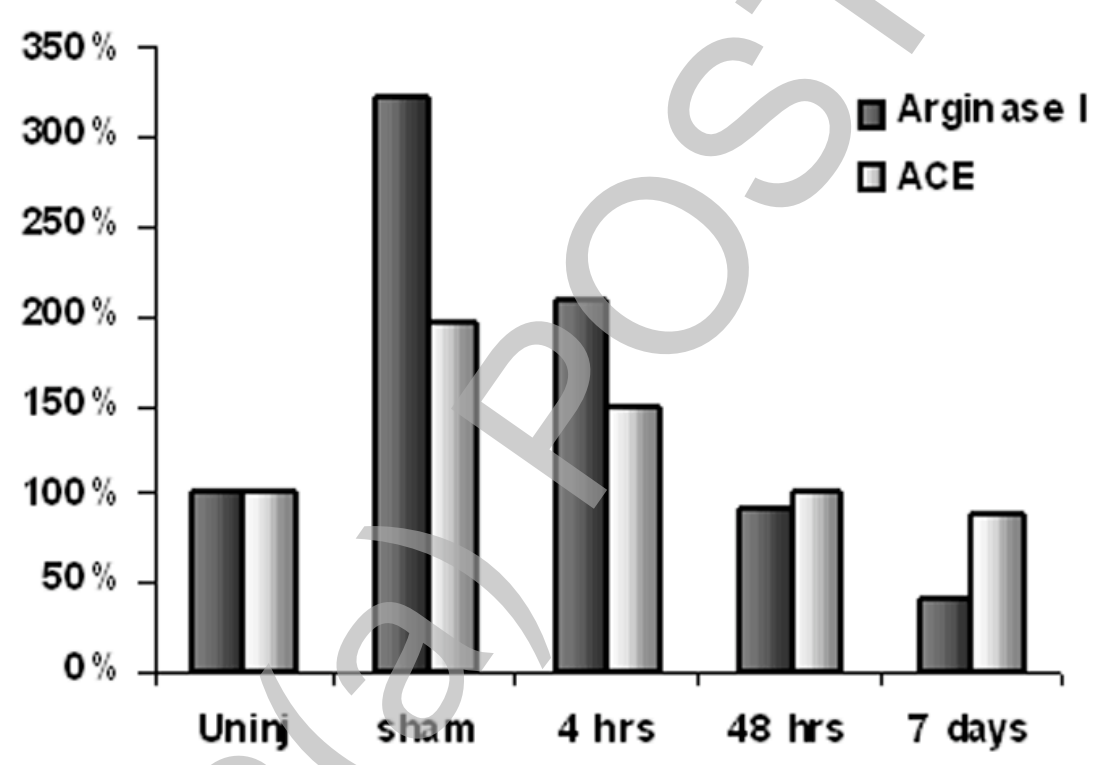

